\section{Type, course and outcome of community acquired infections in hospitalized diabetics}

\author{
Eirini Tsakiridou, ${ }^{1}$ Konstantinos \\ Argyriou, ${ }^{2}$ Demosthenes Makris,' \\ Epameinondas Zakynthinos'
}

'Dept. Critical Care Medicine, University Hospital, Medical School, University of Larisa, Larisa; ${ }^{2}$ General District Hospital of Serres, Greece

\begin{abstract}
Diabetes mellitus has been associated with increased frequency of serious infections which are attributed to immune deficiencies. The aims of this study were to investigate the type, course and outcomes of community acquired infections, and especially bacteremia in diabetics hospitalized for infection. One hundred and thirty-four consecutive patients (67 diabetics and 67 non-diabetics) matched for age, who were admitted to a general District Hospital in Greece due to infection, were included in this case control study. Diabetics presented urinary infections (46.3\% vs. $26.8 \%, \mathrm{P}=0.006)$, skin infections ( $9 \%$ vs. $0 \%, \mathrm{P}=0.007)$ and bacteremia (11.1\% vs. $1.5 \%$, $\mathrm{P}=0.023)$ more often than controls. The most common microorganisms in diabetics were Escherichia coli, Klebsiella pneumoniae, Streptococcus species and fungi. Diabetics had a significantly prolonged hospital stay $(6.7 \pm 5.4$ vs. $4.5 \pm 2.4, \mathrm{P}=0.003$ ) compared to controls. Inhospital mortality was similar in both groups ( $10.4 \%$ vs. $3 \%, \mathrm{P}=0.082)$ but diabetics had an increased risk from death due to bacteremia (Log-odds 4.2, $\mathrm{SE}=1.1, \mathrm{P}<0.0001$ ). Although the analyzed cohorts are small, we found that patients with diabetes mellitus have longer hospitalization related to infections and are at increased risk of bacteremia which may result in adverse outcome.
\end{abstract}

\section{Introduction}

Diabetes mellitus (DM) has been associated with an increased frequency of infections. ${ }^{1,2}$ The occurrence of infection in diabetics may increase the risk for adverse outcomes as underlined in previous studies which demonstrated significant associations between infections and mortality in DM patients..$^{3.5}$ However, despite advances in therapeutic strategies, including the introduction of novel pharmaceutic agents and therapeutic measures, morbidity indices in diabetics are still considerable. ${ }^{12} \mathrm{DM}$ patients are considered to be susceptible to various infections due to impairment of immune function. ${ }^{6-8}$ Thus, even immunocompetent patients with DM may experience an increased risk of several types of infections, presumably depending on the immunosuppressive character of diabetes and on underlying pathophysiology. ${ }^{9-11}$ In Mediterranean countries, there is an assumption that the management of infections is challenging because of the excessive use of antibiotics and of the prevalence of multidrug resistant bacteria (MDR) ${ }^{12-14}$ which often requires the use of pharmaceutic agents which are potentially toxic..$^{15,16}$ In other countries, community acquired bacteria resistant to antibiotics are already considered a major public concern. ${ }^{17}$ However, published data regarding the type of infections due to MDR, especially of a serious nature such as bacteremia, are scarce.

In the present study, we aimed to investigate the type, course and outcomes of community acquired infections, especially bacteremia in diabetic medical patients.

\section{Materials and Methods}

This was an observational case-control study. Diabetic patients admitted to the General district hospital of Serres in Northern Greece between 2000 and 2006 were included in the study if they fulfilled the following criteria: a) over 18 years of age; b) DM type I or type II; and c) admission due to infection. Exclusion criteria were: d) immunosuppression due to cancer or to immunosuppressive agents; e) patients with congenital abnormalities; f) recent admission requiring surgery; g) surgery involving respiratory and urinary system; h) coexistence of unstable disease (other than DM or infection) which required admission. An equal number of age-matched nondiabetic patients admitted due to infection who fulfilled criteria a), c), d), e), f), g), h) were also included in the study as controls.

\section{Baseline measurements}

At baseline, participants underwent clinical examination and basic radiological and laboratory assessment including full blood count, erythrocyte sedimentation rate (ESR), serum biochemical analysis including glucose levels and C-reactive protein (CRP), and microbiology studies including blood, urine or other type of cultures decided by the physician responsible for treatment. For each patient, a Sepsis-related Organ Failure Assessment (SOFA) score was calculated and type of infection, history including duration of diabetes, co-morbidities and number of previous hospitalizations for infections were recorded. Duration of hospitalization and and in-hospital morbidity were estimated at the end of hospitalization/ discharge.
Correspondence: Demosthenes Makris, University of Larisa, Medical School, Larisa, Greece. E-mail: dimomakris@med.uth.gr or appollon7@ hotmail.com

Key words: infection, diabetes mellitus, bacteremia.

Conflict of interest: the authors report no conflicts of interest.

Received for publication: 2 May 2010.

Revision received: 9 September 2010.

Accepted for publication: 10 September 2010.

This work is licensed under a Creative Commons Attribution 3.0 License (by-nc 3.0).

(C) Copyright E. Tsakiridou et al., 2010

Microbiology Research 2010; 1:e6

doi:10.4081/mr.2010.6

\section{Definitions}

Infection and bacteremia were defined according to published guidelines. ${ }^{18} \mathrm{DM}$ was defined according to American Diabetes Association (ADA) criteria. $^{19}$ Bacteremia was confirmed by positive blood cultures. Blood and urine cultures were considered as positive according to the local protocol ( $\left.>10^{5} \mathrm{cfu} / \mathrm{mL}\right)$. SOFA score was used for clinical outcome assessment, as it is commonly used for the prognosis of mortality during the first seven days of hospitalization in critical care patients. ${ }^{20,21}$ For the course of infection, daily mean of WBC and temperature available for each patient were recorded. Febrile was defined by an armpit temperature of over $38^{\circ} \mathrm{C}$ (fever) and patients were considered to be afebrile when armpit temperature remained below $37^{\circ} \mathrm{C}$ for longer than $24 \mathrm{~h}$. Active infection was considered as WBC levels greater than $10,000 / \mathrm{mm}^{3}$. In-hospital morbidity and length of hospital stay were recorded for all patients.

\section{Statistical analysis}

Data are presented as frequency (\%) for qualitative parameters or mean \pm SD for quantitative variables. Comparisons between cases and controls were performed by using the t-test or the $\chi^{2}$ test as appropriate; a $P$ value below 0.05 was considered statistically significant. Univariate and multivariate logistic regression analysis were performed to determine variables associated with bacteremia, in-hospital stay and adverse outcome. The following variables were included in the univariate analysis: sex, age, cardiovascular complications, hypertension, hyperlipidemia, COPD, hypothyroidism and SOFA score on admission. Statistical software SPSS version 17 was used for data analysis. 


\section{Results}

A total of 134 subjects were included in the study made up of 67 patients and 67 controls. Table 1 summarizes baseline characteristics of the study population. Glucose levels in DM patients at admission were $300.12 \pm 153.41$ $\mathrm{mg} / \mathrm{dL}$. Diabetic patients had higher prevalence of cardiovascular disease, hypertension, history of stroke and ESR compared to non-diabetics, and significantly higher frequency of previous hospitalizations due to infection $(\mathrm{P}<0.05)$.

\section{Infections}

Table 2 summarizes all type of infections at admission. Urinary tract infection was the most frequent infection among diabetics and the second most frequent infection among non-diabetics $(\mathrm{P}=0.006)$. Lower respiratory tract infection (35.8\%) was the second most frequent infection in diabetics but was most commonly found (46.3\%) in non-diabetics. The most frequent microorganisms causing urinary tract infections in diabetics and controls were Escherichia coli $(34.5 \% \mathrm{n}=10$ vs. $12.5 \%$ $\mathrm{n}=2 ; \mathrm{P}=0.110)$, fungi $(10.3 \% \mathrm{n}=3$ vs. $0 \%$; $\mathrm{P}=0.183)$, Klebsiella pneumoniae $(6.9 \% \mathrm{n}=2$ vs. $0 \% ; \mathrm{P}=0.283)$ and Proteus mirabilis $(0 \%$ vs. $12.5 \% \mathrm{n}=2 ; \mathrm{P}=0.051$ ).

Skin infections were caused by Streptococcus pyogenes and Staphylococcus aureus and they included cases of erysipelas $(n=1)$, external otitis $(n=2)$ and abscess of lower extremities $(n=1)$.

Table 3 represents 38 microorganisms isolated in biological samples in diabetics and controls. No MDR bacteria were identified.

Overall, bacteremia was found in 8 patients. Bacteremia was significantly more frequent in diabetics compared to controls (7 diabetics vs. one non-diabetic; $\mathrm{P}=0.023)$. Bacteremia was caused by $E$. coli $(\mathrm{n}=1), K$. pneumoniae $(\mathrm{n}=2)$ and Streptococcus pneumoniae $(\mathrm{n}=1)$ in diabetics and Streptococcus viridans $(\mathrm{n}=1)$ in non-diabetics. Univariate analysis did not show any potential risk factor for bacteremia.

\section{Outcomes}

Overall hospital stay was $5.65 \pm 4.31$ days. Diabetics had significantly longer hospital stay (6.7 \pm 5.4 vs. $4.5 \pm 2.4 ; \mathrm{P}=0.003)$ than controls (Table 4). Baseline serum glucose levels in diabetics with hospital stay less than five days were $267.08 \pm 127.99$ vs. $320.26 \pm 165.28$ in those patients with hospital stay over five days $(\mathrm{P}=0.104)$.

Regarding the course of infection, diabetics had a normalization of WBC $\left(<10,000 / \mathrm{mm}^{3}\right)$ and armpit temperature after the $3^{\text {rd }}$ and $7^{\text {th }}$ day of hospital stay, respectively, compared with control subjects for whom these values
Table 1. Baseline characteristics of diabetic patients and controls included in the study.

\begin{tabular}{lccc} 
& $\begin{array}{c}\text { Pts with diabetes mellitus } \\
\mathbf{n}=67\end{array}$ & $\begin{array}{c}\text { Controls } \\
\mathbf{n}=67\end{array}$ & $\mathrm{P}$ \\
Age (years) & $71.4 \pm 12.3(66)$ & $71.4 \pm 13.6(66)$ & 1 \\
Comorbidities & & & \\
Hypertension & $52(80 \%)$ & $36(57.1 \%)$ & 0.005 \\
Hyperlipidemia & $22(33.8 \%)$ & $13(20.6 \%)$ & 0.094 \\
History of stroke & $13(20 \%)$ & $3(4.5 \%)$ & 0.006 \\
Coronary artery disease & $25(37.9 \%)$ & $14(21.2 \%)$ & 0.036 \\
Cardiovascular disease & $4(6.1 \%)$ & 0 & 0.041 \\
Cardiac heart failure & $8(12.1 \%)$ & $4(10.4 \%)$ & 0.760 \\
COPD & $5(7.5 \%)$ & $11(16.4 \%)$ & 0.110 \\
Hypothyroidism & $3(4.5 \%)$ & $1(1.5 \%)$ & 0.310 \\
\hline Hospitalizations/year & $1.2 \pm 0.4$ & $1.05 \pm 0.2$ & 0.017 \\
Total Sofa Score & $1.5 \pm 1.5$ & $1.4 \pm 1.8$ & 0.17 \\
\hline WBC $\left(4-10 \times 10^{9} \mathrm{~L}^{-1}\right)$ & $12950.2 \pm 4830.7$ & $13184.7 \pm 9356.3$ & 0.866 \\
ESR $\left(\leq 20 \mathrm{~mm} \mathrm{~h}^{-1}\right)$ & $77.2 \pm 29.3$ & $49.5 \pm 28.8$ & $<0.001$ \\
\hline CRP $\left(0-0.5 \mathrm{mg}^{\mathrm{d}}\right)$ & $13.3 \pm 8.1$ & $13.2 \pm 6.3$ & 0.967 \\
\hline
\end{tabular}

Data are presented as $n(\%)$ or mean $( \pm S D)$. Differences between patients and controls were evaluated by t-test or $\chi^{2}$ test as appropriate. $\mathrm{COPD}=$ chronic obstructive pulmonary disease.

Table 2. Type of infections on admission.

\begin{tabular}{lccc} 
Type of infection & Pts with diabetes mellitus & Controls & P \\
Urinary tract & $31(46.3 \%)$ & $18(26.9 \%)$ & 0.006 \\
Lower respiratory tract & $24(35.8 \%)$ & $31(46.3 \%)$ & 0.116 \\
\hline Gastrointestinal tract & $5(7.5 \%)$ & $16(23.9 \%)$ & 0.02 \\
Skin & $6(9 \%)$ & $0(0 \%)$ & 0.007 \\
\hline Upper respiratory tract & $1(1.5 \%)$ & $1(1.5 \%)$ & 1 \\
Endocarditis & $0(0 \%)$ & $1(1.5 \%)$ & 0.315 \\
\hline Total & $67(100 \%)$ & $67(100 \%)$ & \\
\hline
\end{tabular}

Data are presented as n (\%). Differences between diabetes mellitus patients and controls were evaluated by $\chi^{2}$ test. Gastrointestinal infections included biliary tract infections and gastroenteritis.

Table 3. Microorganisms isolated from diabetic patients and controls.

\begin{tabular}{lccc} 
Type of infection & $\begin{array}{c}\text { Pts with diabetes mellitus } \\
n=67\end{array}$ & $\begin{array}{c}\text { Controls } \\
n=67\end{array}$ & P \\
Escherichia coli & $10(43.5 \%)$ & $6(40 \%)$ & 0.287 \\
Klebsiella pneumoniae & $3(13 \%)$ & $0(0 \%)$ & 0.08 \\
\hline Fungi & $3(13 \%)$ & $0(0 \%)$ & 0.08 \\
Streptococcus spp. & $3(13 \%)$ & $5(33.3 \%)$ & 0.466 \\
\hline Proteus mirabilis & $0(0 \%)$ & $2(13.3 \%)$ & 0.154 \\
Staphylococcus aureus & $2(8.7 \%)$ & $0(0 \%)$ & 0.154 \\
\hline Pseudomonas aeruginosa & $0(0 \%)$ & $1(6.7 \%)$ & 0.315 \\
Pneumocysti carini & $0(0 \%)$ & $1(6.7 \%)$ & 0.315 \\
\hline Providenchia stuarti & $1(4.3 \%)$ & $0(0 \%)$ & 0.315 \\
Multimicrobial & $1(4.3 \%)$ & $0(0 \%)$ & 0.315 \\
\hline Total & $23(100 \%)$ & $15(100 \%)$ & \\
\hline
\end{tabular}

Data are presented as n (\%). Multimicrobial infections included one case with Pseudomonas aeruginosa, Klebsiella pneumoniae and Proteus mirabilis. Fungi included Candida spp. Differences between DM patients and controls were evaluated by $\chi^{2}$ test.

Table 4. Outcomes of the study.

\begin{tabular}{lccc} 
Type of infection & $\begin{array}{c}\text { Pts with diabetes mellitus } \\
\mathbf{n}=67\end{array}$ & $\begin{array}{c}\text { Controls } \\
\mathbf{n}=67\end{array}$ & $\mathrm{P}$ \\
Frequency of bacteremia & $7(11.1 \%)$ & $1(1.5 \%)$ & 0.023 \\
Hospital stay (days) & $6.7 \pm 5.4(66)$ & $4.5 \pm 2.4(63)$ & 0.003 \\
\hline In-hospital mortality & $7(10.4 \%)$ & $2(3 \%)$ & 0.084 \\
\hline
\end{tabular}

Data are presented as $\mathrm{n}(\%)$ or mean $( \pm \mathrm{SD})$. Differences between DM patients and controls were evaluated by t-test or $\chi^{2}$ test as appropriate. 
were obtained after the $3^{\text {rd }}$ and $6^{\text {th }}$ day of hospital stay, respectively (Figure 1). However, the differences between total WBC $(\mathrm{P}=0.425)$ and temperature $(\mathrm{P}=0.853)$ at day 4 were not significant. On the other hand, there was no normalization of blood glucose levels in diabetic patients during their hospital stay (Figure 2).

There were 9 deaths: 7 deaths (5.2\%) occurred in diabetics and $2(1.5 \%)$ in controls $(\mathrm{P}=0.084)$. In diabetics, 5 out of 7 bacteremia cases in our study were fatal due to septic shock $(n=2)$ and acute distress syndrome $(n=2)$ from lower respiratory tract infection, and only in one case due to septic shock after urinary tract infection. The critical condition of these patients, and the unexpected rapid progress of their disease (mean hospital stay one day), probably meant it was not possible to isolate the corresponding pathogens in all of the cases (K. pneumoniae $\mathrm{n}=1, S$. pneumoniae $\mathrm{n}=1$ ). The mean age of these 5 patients was 63 years: 2 of them had an uneventful medical history and the other 3 had cardiovascular complications. Clinical profiles of the 2 fatal cases of the non-diabetic group were similar. Risk analysis showed that bacteremia was the only independent risk factor for in-hospital fatal outcome (Log-odds 4.2, $\mathrm{SE}=1.1$, $\mathrm{P}<0.0001)$. In contrast, no significant relationship was found between the duration of hospital stay and the several clinical and laboratory variables tested, including glucose levels.

\section{Discussion}

The present study showed that diabetic patients admitted due to infection have more frequent hospitalizations than non-diabetics $(\mathrm{P}=0.017)$ and may have significantly longer hospital stay due to infection compared to nondiabetics $(\mathrm{P}=0.003)$. In addition, bacteremia may be significantly more prevalent in DM patients requiring hospitalization compared to non-diabetics. These findings are in agreement with previous studies which showed that bacteremia was more frequent in diabetics. ${ }^{4}$ In addition, the present study showed that bacteremia in diabetic patients may be a significant independent risk factor for fatal hospital outcome.

In this study, we found there was no difference in in-hospital mortality between diabetic and non-diabetic patients admitted due to infection. Other investigators have come to similar conclusions. ${ }^{4}$ In contrast, other retrospective cohort studies, found that diabetes is a factor of increased risk of dying from infectious disease., ${ }^{3,5}$ A plausible explanation for these controversial results may be attributed to differences in study cohorts or to different treatment protocols for glycemic control and infection, which alter not only the outcome but
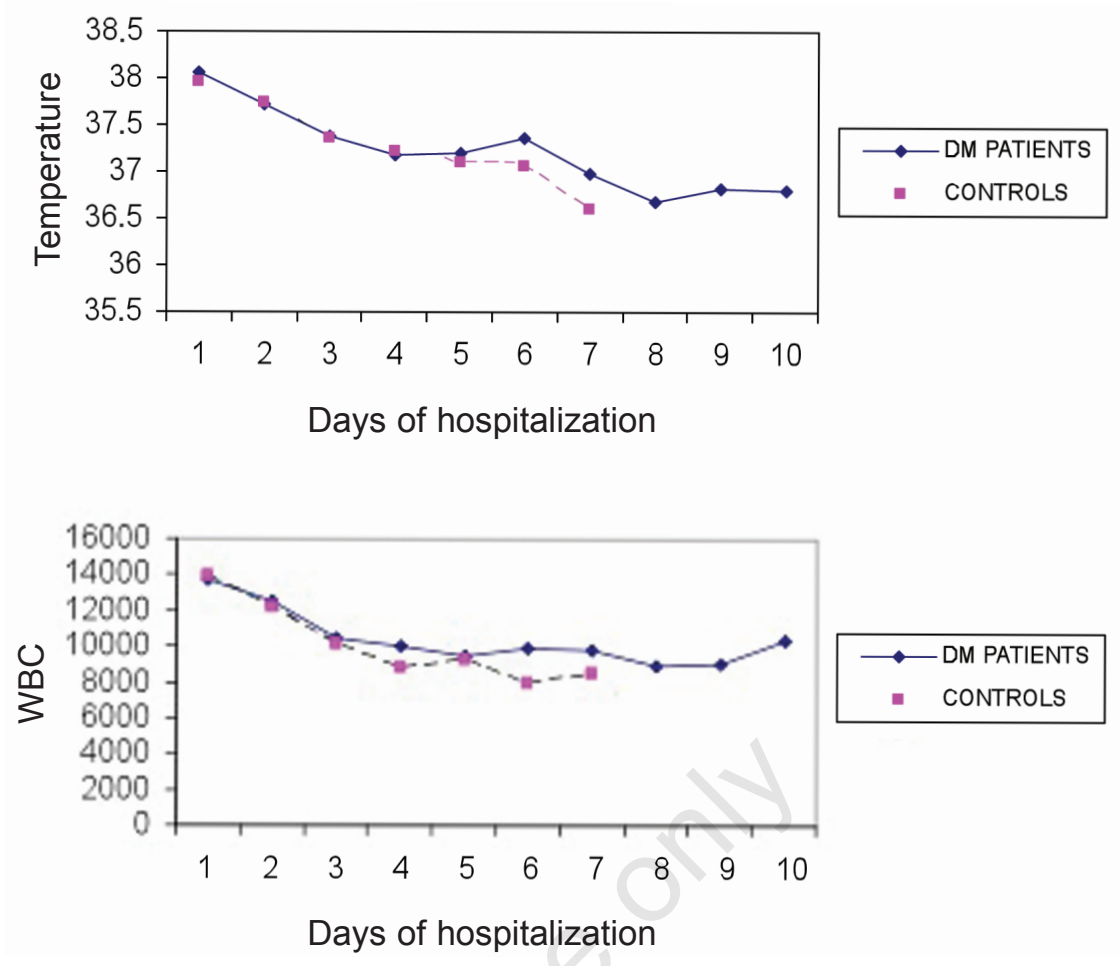

Figure 1. Average temperature $\left({ }^{\circ} \mathrm{C}\right)$ and $\mathrm{WBC}\left(\mathrm{mm}^{3}\right)$ in diabetics and non-diabetics with urinary tract infection.

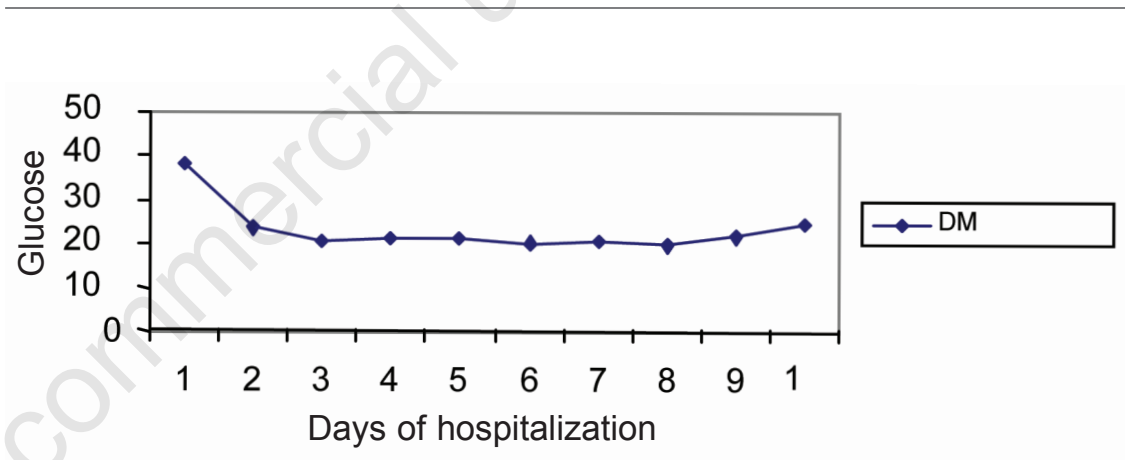

Figure 2. Average blood glucose levels $(\mathrm{mg} / \mathrm{dL})$ in diabetics with urinary tract infection.

also the duration of hospital stay. In addition, in-hospital mortality in diabetics may be attributed to several factors and therefore the contribution of infection to mortality may be underestimated. Diabetic patients experience other significant problems as well, such as cardiovascular or renal disease, which are significant risk factors for increased mortality regardless of the presence of infections.

In the present study, we found an association between DM and prolonged in-hospital stay. Even if we found no significant factor that could independently increase the risk for hospital stay, we observed that there was a short delay in normalization of the temperature levels in diabetics that could be attributed to the patients' immune deficiency. Moreover, considering that diabetic patients had an abnormal mean value of blood glucose levels during their hospitalization, we speculate that, at least in part, diabetic patients may have required longer hospitalization time due to the need for metabolic control, since acute infection may lead to additional stress-related hyperglycemia. ${ }^{22}$ Therefore, this may have additionally contributed to prolonged hospitalization in this study. Similarly, Horcajada and colleagues found that diabetic patients with community acquired urinary tract infection had longer hospitalization compared to nondiabetics. This was attributed to the need to reach an adequate metabolic control or to recover from a more severe infection. ${ }^{23}$

There was a significant difference in erythrocyte sedimentation rate (ESR) between the two groups of patients with infection. As far as other inflammatory indices are concerned, such as C-reactive protein (CRP) and white cells, which are traditionally used as markers of the severity of infection, there was 
no significant difference between patients and controls. ${ }^{24,25}$ ESR may be a good marker reflecting the inflammatory process that occurs during infection. Notably, another study indicated that ESR in diabetics may be elevated in the absence of overt infection. ${ }^{26}$

In our study, bacteremia was significantly more frequent in diabetic patients and was associated with adverse outcome. Bacteremia was attributed to $E$. coli, $K$. pneumoniae and $S$. pneumoniae that were not multiple drugresistant. In this respect, one might argue that these infections could be controlled by using adequate antibiotics. However, despite adequate treatment, 5 out of 7 bacteremia cases in our study were fatal indicating the potential impairment of the diabetic patient's immune response. Nevertheless, this was not investigated in our study and this limitation should be noted.

Regarding the microbiological patterns observed in this study, our findings are in agreement with previous studies which suggested that $E$. coli and $K$. pneumoniae are the most usual microorganisms for infection in diabetics. ${ }^{4,27}$ Additionally, in one of these studies, Stoeckle and colleagues proved that $K$. pneumoniae was the most frequent microorganism causing bacteremia in a group of diabetic patients. This might be due to the fact that DM patients often present urinary infections where the most usual microorganisms of this category of infection are $E$. coli and $K$. pneumoniae. Geerlings and colleagues found that diabetics were more vulnerable in urinary tract infections and that $E$. coli adheres better to uroepithilial cells in DM patients.,28,29

Moreover, according to our study, fungi and specifically Candida species were found to be another leading cause of infections in diabetics, causing exclusively urinary tract infections. However, other studies have come to contrasting conclusions. Diabetes was found to be a risk factor for fungal urinary tract infections by Krcmery and colleagues, whereas González-Pedraza Avilés and colleagues did not found an association between urinary tract infection from Candida and the presence of diabetes. $^{30,31}$

In addition, skin infections in our study were observed only in the group of diabetics and corresponding microorganisms involved in these infections were $S$. aureus and $S$. pyogenes. $S$. aureus has proven to be the most frequent isolated pathogen in another recent study, causing $63 \%$ of soft tissue infections, whereas skin infections were increased in diabetic patients compared with non-diabetics in a Danish general population who participated in the Copenhagen City Heart Study. ${ }^{32,33}$

Moreover, apart from skin infection (one case) in our study Streptococcus was found responsible for 2 pneumonia cases among diabetics. Regarding group B streptococcus infec- tions, Skoff and colleagues found that patients with diabetes were more likely to present with skin and/or soft tissue infections and pneumonia, and that diabetes was present in $44.4 \%$ of all cases, whereas in another study, Schwartz and colleagues observed a 10.5-fold increase in risk of group B streptococcus infections in diabetics. $^{34,35}$

In conclusion, despite the small analyzed cohort, the present study demonstrated that diabetic patients admitted with infection, present bacteremia more often than non-diabetics. In turn, bacteremia, although not caused by MDR bacteria, is a significant factor for increased mortality in diabetic patients. In addition, the present study showed that diabetic patients had longer hospital stay compared to controls. In the light of these findings, rigorous prevention strategies and therapeutic interventions should be implemented in this group of patients, aimed at optimizing metabolic control and early detection of bacteremia by taking a blood culture on admission of all diabetic patients diagnosed with infection. ${ }^{36,37}$

\section{References}

1. Muller LM, Gorter KJ, Hak E, et al. Increased risk of common infections in patients with type 1 and type 2 Diabetes Mellitus. Clin Infect Dis 2005;41:281-8.

2. Patterson JE, Adriole VT. Bacterial urinary tract infections in diabetes. Infect Dis Clin North Am 1997;11:735-51.

3. Shah BR, Hux JE. Quantifying the risk of infectious diseases for people with diabetes. Diabetes Care 2003;26:510-3.

4. Stoeckle M, Kaechb C, Trampuzb A, et al. The role of diabetes mellitus in patients with bloodstream infections. Swiss Med Wkly 2008;138:512-9.

5. Bertoni AG, Saindah S, Brancati FL. Diabetes and the risk of infection-related mortality in the US. Diabetes Care 2001; 24:1044-9.

6. Hopps E, Camera A, Caimi G. Polymorphonuclear leukocytes and diabetes mellitus. Minerva Med 2008;99:197-202.

7. Geerlings SE, Hoepelman AI. Immune dysfunction in patients with diabetes mellitus. FEMS Immunol Med Microbiol 1999; 26:259-65.

8. Calvet HM, Yoshikawa TT. Infections in diabetes. Infect Dis Clin North Am 2001; 15:407-21.

9. Larkin JG, Frie BM, Ireland JT. Diabetes mellitus and infection. Postgrad Med J 1985;61:233-7.

10. Gupta S, Koirala J, Khardori R, et al. Infections in diabetes mellitus and hyperglycemia. Infect Dis Clin North Am 2007; 21:617-38.
11. Nicolle LE. Urinary tract infections in diabetes. Curr Opin Infect Dis 2005;18:49-53.

12. Nseir S, Ader F. Prevalence and outcome of severe chronic obstructive pulmonary disease exacerbations caused by multidrugresistant bacteria. Curr Opin Pulm Med 2008;14:95-100.

13. Nseir S, Di Pompeo C, Diarra M, et al. Relationship between immunosuppression and intensive care unit-acquired multidrug-resistant bacteria: a case-control study. Crit Care Med 2007;35:1318-23.

14. Nseir S, Ader F, Marquette CH, Durocher A. [Impact of fluoroquinolone use on multidrug-resistant bacteria emergence]. Pathol Biol (Paris) 2005;53:470-5.

15. Mentzelopoulos SD, Pratikaki M, Platsouka E, et al. Prolonged use of carbapenems and colistin predisposes to ventilatorassociated pneumonia by pandrug-resistant Pseudomonas aeruginosa. Intensive Care Med 2007;33:1524-32.

16. Michalopoulos AS, Tsiodras S, Rellos K, et al. Colistin treatment in patients with ICUacquired infections caused by multiresistant Gram-negative bacteria: the renaissance of an old antibiotic. Clin Microbiol Infect 2005;11:115-21.

17. Datta R, Huang SS. Risk of infection and death due to methicillin-resistant Staphylococcus aureus in long-term carriers. Clin Infect Dis 2008;47:176-81.

18. Joint Commission on the Accreditation of Healthcare Organizations. Accreditation manual for hospitals. In: Joint Commission on the Accreditation of Healthcare Organizations, ed. Chicago, IL: Joint Commission on the Accreditation of Healthcare Organizations 1994:121-40.

19. Diagnosis and classification of diabetes mellitus. American Diabetes Association (ADA). Diabetes Care 2006;29:S43-48.

20. Vincent JL, Moreno R, Takala J, et al. The SOFA (Sepsis-related Organ Failure Assessment) score to describe organ dysfunction/failure. Intensive Care Med 1996; 22:707-10.

21. Ferreira FL, Bota DP, Bross A, et al. Serial Evaluation of the Sofa Score to Predict Outcome in Critically Ill Patients. JAMA 2001;286:1754-8.

22. Henderson WR, Chittock DR, Dhingra VK, et al. Hyperglycemia in acutely ill emergency patients - Cause or effect? CJEM 2006;8:339-43.

23. Horcajada JP, Moreno I, Velasco M, et al. Community-acquired febrile urinary tract infection in diabetics could deserve a different management: a case-control study. J Intern Med 2003;254:280-6.

24. Bircan A, Kaya 0, Gökirmak M, et al. Creactive protein, leukocyte count and ESR in the assessment of severity of community-acquired pneumonia. Tuberk Toraks 
2006;54:22-9.

25. Brigden ML. Clinical utility of the erythrocyte sedimentation rate. Am Fam Physician 1999;60:1443-50.

26. Elias AN, Domurat E. Erythrocyte sedimentation rate in diabetic patients: relationship to glycosylated hemoglobin and serum proteins. J Med 1989;20:297-302.

27. Thomsen RW, Hundborg HH, Lervang $\mathrm{HH}$ et al. Diabetes mellitus as a risk and prognostic factor for community-acquired bacteremia due to enterobacteria: a 10-year, population-based study among adults. Clin Infect Dis 2005;40:628-31.

28. Geerlings SE, Meiland R, Hoepelman AI. Pathogenesis of bacteriuria in women with diabetes mellitus. Int $\mathbf{J}$ Antimicrob Agents 2002;19:539-45.

29. Geerlings SE, Meiland R, van Lith EC, et al. Adherence of type 1-fimbriated Esche- richia coli to uroepithelial cells: more in diabetic women than in control subjects. Diabetes Care 2002;25:1405-9.

30. Krcmery S, Dubrava M, Krcmery V Jr. Fungal urinary tract infections in patients at risk. Int J Antimicrob Agents. 1999;11: 289-91.

31. González-Pedraza Avilés A, Luís Hernán$\operatorname{dez}$ R, Luna Avila J, et al. Urinary tract infection by Candida species. Aten Primaria 2006;38:147-53.

32. Benfield T, Jensen JS, Nordestgaard BG. Influence of diabetes and hyperglycaemia on infectious disease hospitalisation and outcome. Diabetologia 2007;50:549-54.

33. Masoodi SR, Wani AI, Misgar RA, et al. Pattern of infections in patients with diabetes mellitus-Data from a tertiary care medical centre in Indian sub-continent. Diabetes and Metabolic Syndrome:
Clinical Research and Reviews 2007;1:915.

34. Skoff TH, Farley MM, Petit S, et al. Increasing burden of invasive group $B$ streptococcal disease in nonpregnant adults, 1990-2007. Clin Infect Dis 2009;49: 85-92.

35. Schwartz B, Schuchat A, Oxtoby MJ, et al. Invasive group B streptococcal disease in adults. A population-based study in metropolitan Atlanta. JAMA 1991;266:1112-4.

36. Stolar MW, Hoogwerf BJ, Gorshow SM, et al.. Managing type 2 diabetes going beyond glycemic control. J Manag Care Pharm 2008;14:s2-19.

37. Wolfsthal SD, Manno R, Fontanilla E. Emergencies in diabetic patients in the primary care setting. Prim Care 2006;33: 711-25. 